ESJ Natural/Life/Medical Sciences

\title{
Facteurs Associes Aux Deces Maternels A L'hôpital De Zone Saint Jean De Dieu De Tanguieta De 2015 A 2019
}

\author{
Atade Sèdjro Raoul \\ UER en gynécologie Obstétrique, Institut de Formation en Soins Infirmiers \\ et Obstétricaux (IFSIO), Université de Parakou, Bénin \\ Hounkponou Ahouingnan Fanny Maryline Nouessèwa \\ Obossou Achille Awadé Afoukou \\ UER en gynécologie Obstétrique, Faculté de Médecine, Université de \\ Parakou, Bénin \\ Gabkika Bray Madoué \\ Hôpital du district du sud Ndjamena, Tchad \\ Doha Sèna Mireille Isabelle \\ Sidi Rachidi Imorou \\ Vodouhe Mahublo Vinadou \\ Salifou Kabibou \\ UER en gynécologie Obstétrique, Faculté de Médecine, Université de \\ Parakou, Bénin
}

Doi:10.19044/esj.2021.v17n29p93

Submitted: 18 June 2021

Accepted: 20 July 2021

Published: 31 August 2021
Copyright 2021 Author(s)

Under Creative Commons BY-NC-ND 4.0 OPEN ACCESS

Cite As:

Atade S.R., Hounkponou Ahouingnan F.M.N., Obossou A.A.A., Gabkika B.M., Doha S.M.I., Sidi R.I., Vodouhe M.V. \& Salifou K. (2021). Facteurs Associes Aux Deces Maternels A L'hôpital De Zone Saint Jean De Dieu De Tanguieta De 2015 A 2019. European Scientific Journal, ESJ, 17(29), 93.

https://doi.org/10.19044/esj.2021.v17n29p93

Résumé

Introduction: La mortalité maternelle est un problème de santé publique au Bénin ; Elle est estimée à 397 pour 100000 Naissances Vivantes (NV) en 2017. Dans les pays à faible revenu le ratio de mortalité maternelle est de 239 pour 100000 Naissances Vivantes (NV).

Objectifs: Identifier les facteurs associés aux décès maternels à l'Hôpital de Zone Saint Jean de Dieu de Tanguiéta de 2015 à 2019.

Méthode d'étude: Il s'est agi d'une étude rétrospective à visée descriptive et analytique. Les dossiers des femmes ont été dépouillés pour col- 
lecter les informations relatives aux variables de l'étude.

Résultat: Durant la période d'étude, nous avons recensé 222 dossiers. Le ratio de mortalité maternelle intra-hospitalière était de 1173 décès pour 100 000 naissances. L’âge moyen des femmes décédées était de 25,4 ans. Les femmes décédées étaient des ménagères dans $72,1 \%$. Plus de la moitié des femmes décédées (55,9\%) n’avaient bénéficié d'aucune consultation prénatale. La référence était le principal mode d'entrée à l'hôpital (64\%). Les causes obstétricales directes des décès étaient dominées par les hémorragies (25,8\%), les troubles hypertensifs (22,8\%) et les infections puerpérales (21,2\%). Les facteurs associés aux décès maternels étaient : le milieu de résidence $(\mathrm{p}=0,004)$, le délai (de 5jours et plus) entre l'apparition des symptômes et l'admission à l'hôpital $(\mathrm{p}=0,019)$, le transport non médicalisé $(p=0,013)$ et le troisième retard $(p<0,001)$.

Conclusion: Le ratio de mortalité maternelle était élevé à l’hôpital de zone Saint Jean de Dieu de Tanguieta. Il importe que des actions soient menées en agissant sur les différents facteurs en vue de réduire la mortalité maternelle dans cet hôpital.

Mots-clés: Mortalité Maternelle, Naissance Vivante, Facteurs Associés, Tanguiéta, Bénin

\section{Factors Associated With Maternal Deaths At The Saint Jean De Dieu Zone Hospital In Tanguieta From 2015 To 2019}

Atade Sèdjro Raoul
UER en gynécologie Obstétrique, Institut de Formation en Soins Infirmiers et Obstétricaux (IFSIO), Université de Parakou, Bénin

Hounkponou Ahouingnan Fanny Maryline Nouessèwa

Obossou Achille Awadé Afoukou

UER en gynécologie Obstétrique, Faculté de Médecine, Université de Parakou, Bénin

Gabkika Bray Madoué

Hôpital du district du sud Ndjamena, Tchad

Doha Sèna Mireille Isabelle

Sidi Rachidi Imorou

Vodouhe Mahublo Vinadou

Salifou Kabibou

UER en gynécologie Obstétrique, Faculté de Médecine, Université de Parakou, Bénin 


\section{Abstract}

Introduction: Maternal mortality is a public health problem in Benin, it is estimated at 397 per 100,000 Live Births (LB) in 2017. In low-income countries the maternal mortality ratio is 239 per 100,000 Live Births (LB).

Objectives: Identify the factors associated with maternal deaths at the Saint Jean de Dieu Zone Hospital in Tanguiéta from 2015 to 2019.

Study Method: This was a retrospective study with a descriptive and analytical aim. Women's records were searched to collect information on study variables.

Result: During the study period, we identified 222 cases. The intrahospital maternal mortality ratio was 1,173 deaths per 100,000 births. The average age of the deceased women was 25.4 years. $72.1 \%$ of the deceased women were housewives. More than half of the women who died (55.9\%) had not received any prenatal consultation. Referral was the main mode of entry to hospital (64\%). The direct obstetric causes of death were dominated by haemorrhages (25.8\%), hypertensive disorders (22.8\%) and puerperal infections (21.2\%). Factors associated with maternal deaths were: place of residence $(p=0.004)$, the time ( 5 days or more) between the onset of symptoms and admission to hospital $(\mathrm{p}=0.019)$, unsafe transportation $(\mathrm{p}=$ 0.013) and The third delay ( $<<0.001)$.

Conclusion: The maternal mortality ratio was high at the Saint Jean de Dieu hospital in Tanguieta. It is important that actions be taken by acting on the various factors in order to reduce maternal mortality in this hospital.

Keywords: Maternal Mortality, Live Birth, Associated Factors, Tanguiéta, Bénin

\section{Introduction}

La mortalité maternelle est un problème de santé publique (Ministère de la santé bénin, 2018). Le ratio de mortalité maternelle était de 239 pour 100 000 Naissances Vivantes (NV) dans les pays à faible revenu contre 12 pour $100000 \mathrm{NV}$ dans les pays à revenu élevé en 2015 (Castillo et al, 2017). La mortalité maternelle en Afrique occupe $57 \%$ de la mortalité maternelle à l'échelle mondiale (Yaya et al, 2017). En Afrique subsaharienne, le taux de mortalité maternelle, reste parmi les plus élevés au monde, avec des chiffres de 542 pour 100000 naissances vivantes en 2017 (OMS, 2019). Au Bénin, le taux de mortalité maternelle est de 397 pour 100000 naissances vivantes en 2017 (OMS, 2019). Pour réduire cette mortalité, le Bénin a pris l'engagement d'œuvrer à la réalisation de l'Objectif de Développement Durable (ODD) numéro 3 ; Cet objectif est de ramener le ratio de mortalité maternelle à moins de 70 pour 100000 NV en 2030 (Alkema et al, 2016). Pour y arriver il convient d'identifier les facteurs sur lesquels il faut agir pour mieux adapter les 
stratégies au contexte local. Le présent travail a pour objectif d'identifier les facteurs associés aux décès maternels à l'hôpital Saint Jean de Dieu de Tanguiéta (HSJD).

\section{Méthode d'étude}

L'étude s'était déroulée dans le service de Gynécologie et d'Obstétrique de l'Hôpital de Zone Saint Jean de Dieu de Tanguiéta.

Il s'agissait d'une étude descriptive et analytique avec recueil rétrospectif des données, qui s'est déroulée sur une période de cinq (05) ans allant du $1^{\mathrm{er}}$ janvier 2015 au 31 décembre 2019. La population d'étude était constituée du dossier de toutes les femmes admises dans le service pour une complication en rapport avec la grossesse, l'accouchement ou le post-partum. Tous les dossiers de femmes reçues pendant la période d'étude pour complication ont été inclus dans l'étude. Pour parler de décès maternel nous avons tenu compte des critères de définition du décès maternel de l'OMS (OMS, 2016). Les dossiers inexploitables ont été exclus de l'étude. Les dossiers de toute femme décédée dans le service en dehors de la grossesse, de l'accouchement ou des suites de couches ou les dossiers de toute femme décédée d'une cause qui n'est pas en rapport avec la grossesse, l'accouchement ou le post-partum n'ont pas été inclus dans l'étude. Il en est de même pour les dossiers de toute femme décédée hors du service et dont le décès est constaté à l'admission. Nous avons procédé à un dépouillement exhaustif des dossiers de toutes les femmes répondant à nos critères d'inclusion durant notre période de collecte. La variable dépendante de l'étude a été le décès maternel; les variables indépendantes ont porté sur les caractéristiques sociodémographiques, les antécédents, le mode d'admission des femmes à l'hôpital, le début de la symptomatologie, les causes du décès maternel et la prise en charge des patientes.

Les données ont été collectées au moyen d'une fiche d'enquête. Une fois la collecte terminée, elles ont été saisies dans le logiciel Epi data 3.1.fr. Le modèle de la régression logistique a été utilisé avec inclusion des variables ayant un seuil de significativité de $20 \%$ en analyse uni variée. La stratégie pas à pas descendante a été utilisée et les variables retenues dans le modèle final étaient celles dont le seuil de significativité était de 5\%.

\section{Résultats}

Durant la période d'étude 227 dossiers ont été dépouillés. De l'année 2015 à l'année 2019, 116 décès maternels ont été enregistrés à l'HSJD de Tanguiéta. Parmi ces cas de décès maternels, 111 ont été inclus dans la présente étude ; 5 autres ont été exclus. 


\section{Ratio de mortalité maternelle à l'hôpital Saint Jean de Dieu de Tanguiéta de 2015 à 2019}

Durant la période d’étude, 116 décès étaient enregistrés pour 9892 naissances vivantes. Soit un ratio de mortalité maternelle intra hospitalière de 1173 pour 100000 naissances vivantes.

En 2015 et 2016, le ratio de mortalité maternelle tournait autour de 900 pour 100000 NV. En 2017, nous avons connu un pic du ratio de la mortalité maternelle allant jusqu’à 1810 pour 100000 NV avant de décroitre les années suivantes jusqu'à 882 pour $100000 \mathrm{NV}$ en 2019. La figure suivante nous montre l'évolution du ratio de mortalité maternelle par année durant la période d'étude.

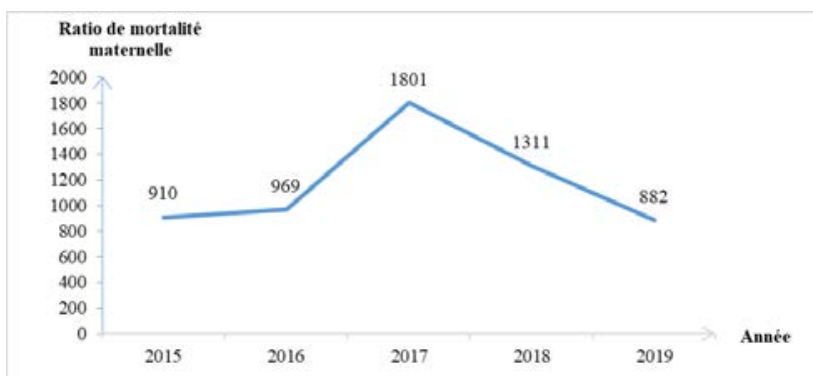

Figure 1. Répartition du ratio de mortalité maternelle de 2015 à 2019 à l’HSJD de Tanguiéta

\section{Caractéristiques sociodémographiques}

La tranche d’âge la plus représentée était celle des femmes âgées de 15 à 24 ans dans une proportion de 53,1\%. Les âges extrêmes étaient de 15 et 48 ans. Les femmes décédées étaient des ménagères $(72,1 \%)$ et vivaient en milieu rural (82,9\%).

Tableau I. Répartition des femmes décédées en fonction des caractéristiques socio démographiques à l'HSJD de Tanguiéta

\begin{tabular}{|c|c|c|}
\hline & Effectif & Fréquence \\
\hline Tranches d'âge (année) & & 53,1 \\
\hline $15-24$ & 59 & 37,8 \\
\hline $25-34$ & 42 & 9,1 \\
\hline$>35$ & 10 & 11,7 \\
\hline Profession & & 4,5 \\
\hline Artisan & 13 & 72,1 \\
\hline Commerçante /Revendeuse & 5 & 1,8 \\
\hline Ménagère & 80 & 2,7 \\
\hline Fonctionnaire & 2 & 7,2 \\
\hline Etudiante/élève & 3 & 82,9 \\
\hline Autre & 8 & 17,1 \\
\hline Résidence & & \\
\hline Rural & 92 & \\
\hline Urbain & 19 & \\
\hline
\end{tabular}




\section{Caractéristiques liées à la référence}

La majorité des femmes décédées (66\%) venaient à l'hôpital entre le $1^{\mathrm{er}}$ et le 4 ème jour après le début de la symptomatologie. Certaines d'entre elles (34\%) consultaient 5 jours après ou même plus. La référence était le principal mode d'entrée (64\%) à l'hôpital. Le moyen de transport utilisé par les femmes était l'ambulance $(59,2 \%)$, la moto $(34,8 \%)$ et la voiture personnelle (6\%).

\section{Caractéristiques liées aux consultations prénatales}

Plus de la moitié des femmes décédées (55,9\%) n’avaient bénéficié d'aucune consultation prénatale (CPN) au cours de leur grossesse. Celles ayant réalisé $4 \mathrm{CPN}$ ou plus ne représentaient que $2 \%$ de l'échantillon.

\section{Caractéristiques liées aux antécédents obstétricaux}

Les primigestes (Première grossesse) étaient les plus représentées (30,7 \%). La gestité moyenne était de 3,6. Quant à la parité, les nullipares (Celles qui n’ont jamais accouché) étaient les plus représentées (26,1\%) ; la parité moyenne était de 2,7. Le tableau suivant résume les antécédents obstétricaux des patientes décédées.

Tableau II.Répartition des femmes décédées à l’Hôpital Saint Jean de Dieu de Tanguiéta en fonction de leurs antécédents obstétricaux

\begin{tabular}{|c|c|c|}
\hline & Effectif & Pourcentage \\
\hline Gestité & & \\
\hline Primigeste (1) & 34 & 30,7 \\
\hline Paucigeste (2-3) & 24 & 21,6 \\
\hline Multigeste (4-5) & 27 & 24,3 \\
\hline Grande multigeste ( $\geq 6)$ & 26 & 23,4 \\
\hline Parité & & \\
\hline Nullipare (0) & 29 & 26,1 \\
\hline Primipare (1) & 23 & 20,7 \\
\hline Paucipare (2-3) & 20 & 18,0 \\
\hline Multipare (4-5) & 22 & 19,9 \\
\hline Grande Multipare $(\geq 6)$ & 17 & 15,3 \\
\hline
\end{tabular}

\section{Caractéristiques liées à la prise en charge}

Certaines femmes décédées ont été prises en charge (27,9\%) sans se référer au protocole de prise en charge des urgences établi dans le service. De plus le troisième retard a été identifié dans $33,3 \%$ des cas. 


\section{Caractéristiques relatives aux décès maternels \\ - Période de survenue de décès}

Plus de la moitié des décès des femmes était survenue en suite de couches (54,1\%). Les autres décès avaient eu lieu pendant la grossesse $(27,9 \%)$, le post partum (14,44\%) et pendant l'accouchement (3,6\%).

\section{- Délai entre l'admission de la patiente à l'hôpital et le décès}

Après l'admission des patientes, le décès était survenu dans 10,8\% avant 1 heure. Entre 1 heure et 24 heures, 44,1\% des patientes admises étaient décédées. Le pourcentage de décès après 24 heures était de 45,1\%.

\section{- Causes de décès maternel}

Parmi les 111 cas de décès maternel, 66 (59,5\%) étaient dus à des causes obstétricales directes contre $48(40,5 \%)$ pour les causes obstétricales indirectes. Les causes obstétricales directes étaient principalement l'hémorragie $(n=17 ; 25,8 \%)$; les troubles hypertensifs $(n=15 ; 22,8 \%)$ et les infections puerpérales $(\mathrm{n}=14 ; 21,2 \%)$. L’anémie $(\mathrm{n}=16 ; 35,3 \%)$ était la principale cause obstétricale indirecte de décès maternel.

\section{Facteurs associés au décès maternel}

L’âge $(\mathrm{p}=0,952)$, et la profession $(\mathrm{p}=0,528)$ n'étaient pas associés au décès maternel. La non réalisation des consultations prénatales n'était également pas associée au décès maternel $(\mathrm{p}=0,375)$. De même il n'existait pas de lien statistiquement significatif entre les antécédents obstétricaux et la mortalité maternelle $(\mathrm{p}=0,065)$.

Le milieu de résidence par contre était significativement associé au décès maternel avec $p=0,018$. En effet les femmes enceintes étaient 2,4 fois plus exposées au décès maternel en milieu rural qu'en milieu urbain $(\mathrm{OR}=$ 2,4 ; IC 95\% [1,2 - 4,8]).

Le délai de 5 jours et plus entre le début de la symptomatologie et l'arrivée à l'hôpital $(\mathrm{p}=0,003)$, le type de transport $(\mathrm{p}=0,013)$ étaient statistiquement liés au décès maternel. En effet, les patientes qui venaient à l'hôpital plus de 4 jours après le début des symptômes étaient 3 fois plus susceptibles de décéder que celles qui venaient avant 4 jours ( $\mathrm{OR}=3$; IC $95 \%:[1,3-6,7])$.

De même, les patientes ayant bénéficié d'un transport médicalisé couraient moins le risque de décéder que ceux qui n’en avaient pas bénéficié $(\mathrm{OR}=0,3$; IC $95 \%$ : $[0,1-0,8])$.

Le troisième retard $(\mathrm{p}<0,001)$ était associé au décès maternel ; Les femmes chez qui le troisième retard avait été noté couraient 11 fois plus de risque de décéder que les femmes chez qui aucun retard n’avait été observé $(\mathrm{OR}=11,3$; IC 95\% : [3,5-6,9]) 
Tableau III. Répartition des femmes décédées à l’Hopital Saint Jean de Dieu de Tanguiéta en fonction du milieu de résidence, du délai d'arrivée à l’hôpital, du mode d’admission, du type de transport et du troisième retard

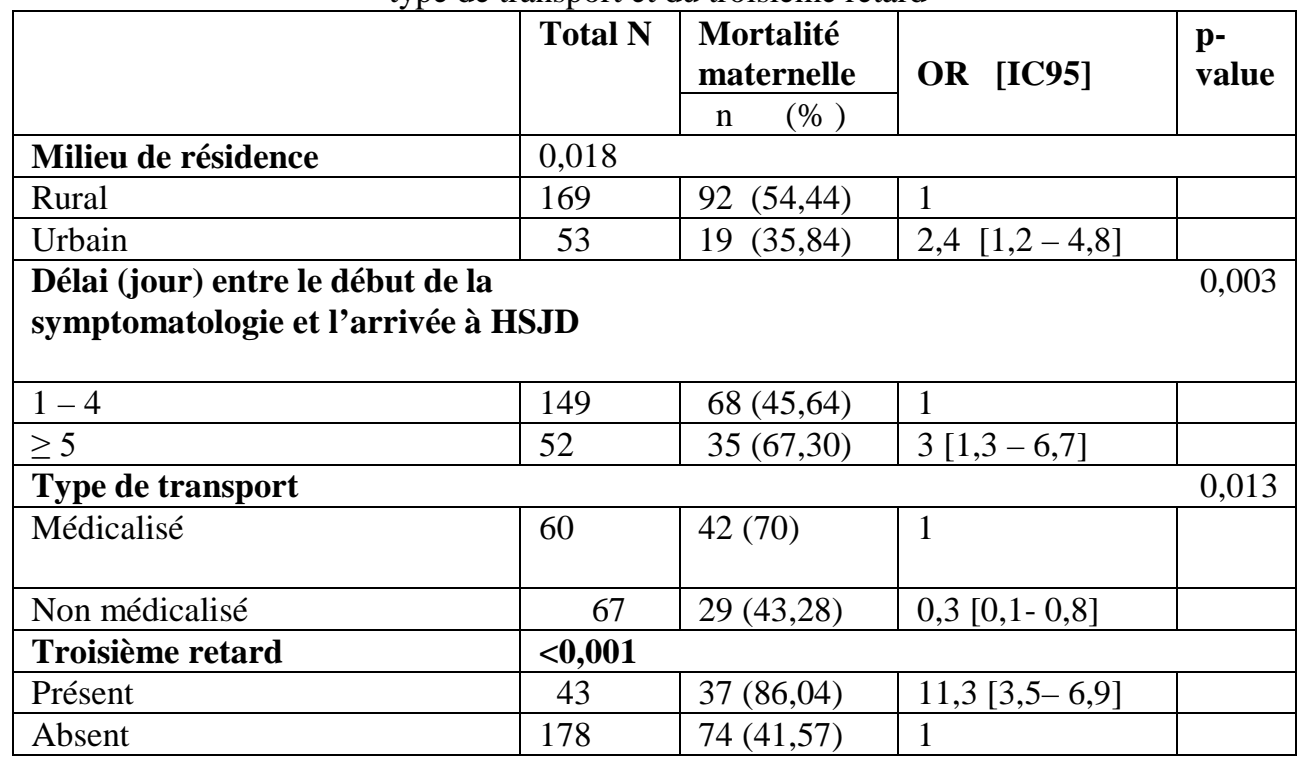

\section{Discussion}

\section{Ratio de la mortalité maternelle}

Le ratio de mortalité maternelle enregistré au cours des 05 années était de 1173 pour 100000 naissances vivantes. Une étude réalisée au centre hospitalier régional du Niger avait observé un ratio similaire, qui était de 1198 pour 100000 naissances vivantes (Ibrahim et al., 2006). Des ratios largement supérieurs au nôtre avaient été enregistrés par plusieurs auteurs dans différents pays ; En Guinée en 2016 lors d’une étude rétrospective portant sur la mortalité maternelle, le ratio de mortalité maternelle était de 1944 pour 100 000 NV (Balde et al., 2016). Au Niger en 2018, le ratio était de 2512 pour 100 000 NV (Alkassoum et al., 2018). Tous ces ratios retrouvés sont nettement plus élevés que ceux retrouvés en 2017 en Afrique subsaharienne (542 pour $100000 \mathrm{NV}$ ), en Afrique du nord et en Océanie respectivement 100 et 229 pour $100000 \mathrm{NV}$ et dans le monde 211 pour $100000 \mathrm{NV}$ (OMS 2019).

Indicateur très important de la santé, le ratio de mortalité maternelle dans le monde présente un contraste criard entre pays développés et pays en voie de développement. Au Bénin les spécialistes sont en nombre insuffisant. L'hôpital de zone Saint Jean de Dieu de Tanguieta a deux médecins gynécologues pour 273089 habitants; Ils se font aider par des médecins généralités dits à ' 'compétence chirurgicale'" qui ne maitrisent pas toujours les protocoles de prise en charge des urgences obstétricales. Or le troisième retard ( $\mathrm{p}<0,001)$ est un facteur non négligeable dans la survenue des décès maternels. Le recrutement de spécialiste pourrait donc réduire les décès 
maternels liés à une prise en charge non adaptée. En outre, à Tanguieta les femmes décédées ont consulté tardivement ; Un délai de plus 4 jours entre le début de la symptomatologie et le recours aux soins a été observé et le délai de plus de 4 jours était statistiquement lié au décès maternel. Il importe que des séances de sensibilisation soient réalisées afin de mettre un accent sur la nécessité de consulter précocement en cas d'anomalie.

\section{Caractéristiques sociodémographiques}

La tranche d'âge la plus représentée de notre étude était celle de 15 à 24 ans. Une étude réalisée au Niger sur la mortalité maternelle intrahospitalière retrouvait un âge moyen de 26 ans (Alkassoum et al., 2018). La tranche d'âge de 15 à 24 ans retrouvée à l'Hôpital Saint Jean de Dieu s'expliquerait par les mariages précoces qui sont fréquents à Tanguiéta. Selon une enquête démographique de santé (INSAE, 2018) réalisée en 2018 au Bénin, les adolescentes contribuent à $21 \%$ de la fécondité. Cette étude retrouve également que $15 \%$ des décès liés aux avortements sont retrouvés dans la tranche d'âge de 15 à 19 ans.

En ce qui concerne la profession, 72,1\% des femmes décédées étaient des ménagères. Ce résultat est comparable aux données rapportées dans une étude réalisée à l'hôpital régional de Labé où $73 \%$ des femmes décédées étaient des ménagères (Balde et al., 2020). En effet, ce sont des femmes au foyer non salariées (Nayama et al., 2006) et dont la charge financière repose le plus souvent sur leur conjoint. Ainsi lorsqu'une pathologie survient, elles sont obligées d'attendre leur époux avant de se rendre dans les formations sanitaires pour être prises en charge ; Ce qui expliquerait le délai souvent long entre le début de la symptomatologie et le recours aux soins.

\section{Caractéristiques liées à la référence}

Le principal mode d'entrée à l'hôpital dans cette étude était la référence. En effet, $64 \%$ des cas avaient été référés. Un taux similaire de référence parmi les cas de décès maternels avait été rapporté à l'hôpital régional de Kayes ; Il était de 70\% (Diassana et al., 2020). Ce sont les femmes référées qui décèdent le plus souvent et ceci s'explique par un retard à l'évacuation des gestantes (Mayi-tsonga et al., 2010).

En ce qui concerne le moyen de référence $59,2 \%$ des patientes avaient bénéficié d'une référence par ambulance. Même constat fait au Niger en 2018 où $52,5 \%$ des cas décédés avaient bénéficié d'un transport par ambulance (Alkassoum et al., 2018). Ce taux élevé de référence par ambulance pourrait s'expliquer par "l'initiative femme pour femme » instaurée dans les zones sanitaires de l'Atacora, qui stipule que chaque femme enceinte qui cotise 200 Francs CFA à une Consultation prénatale bénéficiera d'un transfert gratuit par ambulance lors d'une référence. Dans la présente étude, le type de transport 
lors des références avait influencé la survenue de décès maternels $(\mathrm{p}=0,013)$. Cette initiative femme pour femme a permis de réduire les décès maternels liés à un défaut de transport car les femmes référées arrivaient au centre dans un délai très court par voie de route.

\section{Caractéristiques liées aux paramètres de suivi de la grossesse}

S'agissant des CPN, il y avait 55,9\% des femmes qui n'en avaient pas bénéficié au cours de leur grossesse. Les audits de décès maternels à l'hôpital régional de Kayes aboutissaient aux mêmes conclusions : 50\% des patientes décédées n’avaient réalisé aucune CPN (Diassana et al., 2020). Ceci pourrait s'expliquer par l'ignorance de l'importance des consultations prénatales. Il n’y avait pas d'association significative entre l'absence de CPN et la survenue de décès maternel dans notre étude $(\mathrm{p}=0,375)$. Même si la consultation prénatale ne permet pas de prévenir tous les risques, la CPN bien faite permet de repérer les femmes aux antécédents majeurs pour leur orientation à temps dans une structure de référence.

\section{Causes obstétricales directes de décès maternel}

Les causes obstétricales directes étaient majoritairement responsables de 59,5\% des décès dans notre étude. Cette proportion est inférieure à celle retrouvée au Tchad qui était de 75,7 \% (Foumsou et al., 2014). A l'hôpital Saint Jean de Dieu de Tanguiéta, les causes directes de décès maternel étaient principalement représentées par les hémorragies $(25,8 \%)$, les troubles hypertensifs (22,8\%) et les infections (21,2\%).

L'hémorragie a constitué la principale cause directe de décès au Burkina Faso dans 34,3\% (Ouattara et al., 2017) et au Niger dans 25,5\% (Alkassoum et al., 2018). L'hémorragie en particulier celle du post-partum immédiat est une urgence qui nécessite une intervention immédiate et appropriée. Elle nécessite souvent des actions coordonnées pour une prise en charge précoce, méthodique et adaptée, donc une organisation efficace et permanente du système sanitaire. Cela implique des ressources matérielles immédiatement accessibles, des produits sanguins disponibles et un personnel compétent et dynamique car tout retard ou toute improvisation pourraient concourir à une aggravation du pronostic maternel.

Les troubles hypertensifs constituaient la deuxième cause directe de mortalité maternelle avec une proportion de 22,8\% à Tanguiéta. En Tanzanie il avait été rapporté que les troubles hypertensifs étaient la première cause de décès maternel avec une proportion de 19,9\% (Pembe et al., 2014). Même fait au Mali où les troubles hypertensifs dans 38\% constituaient la première cause directe de décès maternel (Diassana et al., 2020). Les progrès de la réanimation et l'intégration du sulfate de magnésium dans la prise en charge des 
hypertensions artérielles ont permis de réduire les issues défavorables des troubles hypertensifs associés à la grossesse.

\section{Facteurs associés aux décès maternels}

Au terme de la présente étude, une analyse multivariée a permis de ressortir les facteurs associés aux décès maternels à l'hôpital Saint Jean de Dieu de Tanguieta. Il s'agit du milieu de résidence $(\mathrm{p}=0,018)$, du délai de 5 jours et plus entre l'apparition des symptômes et l'admission à l'hôpital ( $\mathrm{p}=$ $0,003)$, du transport non médicalisé $(\mathrm{p}=0,013)$ et le troisième retard $(\mathrm{p}<$ 0,001 ). D'autres facteurs associés au décès maternel ont été retrouvés au Congo en 2015 (Yambare et al., 2017). Il s'agit de l'âge de la victime, la multiparité, l'absence de consultation prénatale et le mode d'admission. Les facteurs liés au décès maternel sont en rapport avec les compétences limitées des prestataires, dans le management clinique des urgences obstétricales ; (Mveba et al., 2018); Il s'agit des dysfonctionnements par rapport à l'anticipation sur la disponibilité d'intrants, la fonctionnalité des équipements, les interactions entre les membres de l'équipe de soins, l'identification et la résolution à temps des problèmes. En Ethiopie par contre les auteurs de leur côté parlaient plutôt de facteurs de risque associés au décès maternel (Godefay et al., 2015). Ils retrouvaient que les femmes qui n'étaient pas membres de 'l'Armée de développement des femmes volontaires"' étaient plus susceptibles de connaître la mort maternelle (OR 2,07, IC à 95\% 1,04-4,11), tout comme les femmes dont les maris ou partenaires avaient des scores inférieurs à la médiane pour la participation pendant la grossesse (OR 2,19 IC à 95\% 1,14-4,18). Les femmes ayant des antécédents préexistants d'autres maladies étaient également exposées à un risque accru (OR 5,58, IC à 95\% $2,17-14,30)$, tout comme celles qui n'avaient jamais utilisé de contraceptifs (OR 2,58, IC à 95\% 1,37-4,85). On en déduit que les facteurs associés au décès maternel varient d'une région à une autre d'un système sanitaire à un autre.

\section{Conclusion}

Le ratio de mortalité maternelle reste élevé à l'hôpital de zone Saint Jean de Dieu de Tanguieta. Il est de 1173 décès maternel pour 100000 naissances vivantes. Les femmes décédées étaient jeunes ayant entre 15 et 24 ans. Elles étaient en majorité des ménagères et n'avaient suivi aucune CPN au cours de leur grossesse. Les facteurs associés au décès maternel étaient le milieu de résidence, le délai de 5 jours et plus entre l'apparition des symptômes et l'admission à l'hôpital, le transport non médicalisé et le troisième retard. Il importe que des actions soient menées en agissant sur les différents facteurs en vue de réduire la mortalité maternelle à l'hôpital de zone Saint Jean de Dieu de Tanguieta. 


\section{References:}

1. Ministère de la santé DSME (2018).Plan opérationnel de réduction de la mortalité maternelle et néonatale au Bénin PO-RMMN 2018- 2022 : Bénin, Cotonou Repéré https://www.prb.org/wpcontent/uploads/2020/06/Benin-Plan-Operationnel-de-Reduction-dela-Mortalite-Maternelle-et-Neonatale-au-Benin.pdf

2. Castillo P., Hurtado J.C., Martinez M.J., Jordao D., Lovane L., Ismail M.R. (2017). Validity of a minimally invasive autopsy for cause of death determination in maternal deaths in Mozambique An observational study. PLoS Med, 14(11), 1-15

3. Yaya S., Kadio B. (2017). Risquer la mort pour donner la vie: politiques et programmes de santé maternelle et infantile en Afrique. Québec : Presses de l'Université Laval

4. OMS. (2019). Evolution de la mortalité maternelle 2000-2017: Estimations de l'OMS, de l'UNICEF, de l'UNFPA, du Groupe de la Banque mondiale et de la Division de la population des Nations Unies : Genève Repéré https://apps.who.int/iris/handle/10665/332962.

5. OMS. (2016). Mortalité Maternelle. Aide-mémoire N³48. Repéré www.who.int/mediacentre/factsheets/fs348/fr/.

6. Alkema L., Chou D., Hogan D., Zhang S., Moller A-B., Gemmill A. (2016). Global regional and national levels and trends in maternal mortality between 1990 and 2015, with scenario-based projections to 2030: a systematic analysis by the UN Maternal Mortality Estimation Inter-Agency Group. Lancet . 387, 462-74. doi.org/10.1016/S01406736(15)00838-7

7. Ibrahim S. (2006). Mortalité maternelle au niveau d'une maternité d'un centre hospitalier régional du Niger : Etude rétrospective à propos de 70 cas (Thèse de doctorat en médecine). Niamey : Université Abdou Moumouni ;

8. Balde O., Balde I.S., Diallo M.H., Diallo A.B., Diallo B.S., Donamou S. (2016). Mortalité maternelle à l'hôpital régional de Kindia: facteurs épidémiologiques et axes stratégiques d'intervention. Rev int sc méd. 18(1), 61-65.

9. Nayama M., Djibo-koba A., Garba M., Idi N., Marafa A., Diallo F.Z., ..., Illa-Souley A. (2006) Mortalité maternelle au niveau d'une maternité de référence du Niger : Etude prospective à propos de 104 cas. Médecine d'Afrique Noire. 53(12), 688- 693

10. Alkassoum I., Djibo I., Hama Y., Abdoulwahabou A., Amadou O. (2018). Facteurs de risques de mortalité maternelle intra hospitalière dans la région de Maradi, Niger (2008-2010) : à propos d'une étude rétrospective réalisée dans les 7 maternités régionales. Méd.Santé trop. 28 (1), 86-91. 
11. INSAE. (2018). Cinquième Enquête Démographique et de Santé au Bénin, 2017-2018 : Indicateurs Clés. Cotonou Repéré https://insae.bj/images/docs/insae-statistiques/enquetesrecensements/EDS/2017-2018/1.Benin_EDSBV_Rapport_final.pdf

12. Balde O., Balde I.S., Diallo M.H., Sylla I., Diallo F.B., Diallo A.B. (2020). Décès maternels à la Maternité de l'hôpital régional de Labé (Guinée). Rev int sc med. 22(1), 34-37.

13. Diassana M., Dembele S., Macalou B., Ndaou K., Sidibbe A., Bocoum A. (2020). Audits des décès maternels à l'hôpital regional de Kayes: Place des 3 retards et impacts dans le service de Gynécologie Obstétrique. Health SCI Dis. 21(10), 59-63.

14. Mayi-Tsonga S., Oksana L., Diallo T., Metogho M., Ndombi I., Mounanga M. (2010). Etude sur les décès maternels au centre hospitalier de Libreville. Médecine d'Afrique Noire. 56(8/9), 426-30

15. Foumsou L., Saleh A., kaïmba O., Djongali S., Djimté N., Mignagnal K. (2014). Les Déterminants de la mortalité maternelle de l'Hôpital Général de Référence Nationale de N’Djamena - Tchad. Revue scientifique du Tchad. 1(5), 1-7.

16. Ouattara A., Ouédraogo S., Lankoandé BCN., Kain DP., Tougma SA., Ouédraogo MC. (2017). Causes of Maternal Mortality According to Reports of Maternal Death Audits in the University Teaching Hospital Bogodogo (UTH-B) from January to December 2017. Open Journal of Obstetrics and Gynecology. 8:1345-353.

17. Pembe B.A., Paulo C., D’mello B.S., Van Roosmalen J. (2014). Maternal mortality at Muhimbili National Hospital in Dar-es-Salaam, Tanzania in the year 2011. BMC Pregnancy and Childbirth. 14(320), 1-7

18. Yambare A., Ibemba G. (2017). Analyse des déterminants de la Mortalité maternelle pré partum en République du Congo 2013-2015. [s.l.] : HAL archives-ouvertes

19. Mbeva J.B.K., Karemere H., Prudence M.N., Nyavanda L., Mundama J.P. (2018). Facteurs explicatifs des décès maternels en milieu hospitalier : Une étude au niveau de six zones de santé dans l'EST de la République Démocratique du Congo. International Journal of Innovation and Applied Studies. 23(4), 559-68

20. Godefay H., Byass P., Graham W.J., Kinsman J., Mulugeta A. (2015). Risk Factors for Maternal Mortality in Rural Tigray, Northern Ethiopia: A Case-Control Study. PLoS ONE. 10(12), 1-12 